\title{
Phosphorylated ERK5/BMK1 transiently accumulates within division spindles in mouse oocytes and preimplantation embryos
}

\author{
Zuzanna Maciejewska ${ }^{1}$, Aude Pascal ${ }^{2}$, Jacek Z. Kubiak ${ }^{2}$, Maria A. Ciemerych ${ }^{3}$ \\ ${ }^{1}$ Department of Embryology, Institute of Zoology, Faculty of Biology, University of Warsaw, Poland \\ ${ }^{2}$ Institute of Genetics and Development, CNRS-UMR 6061, Cell Cycle Group, \\ IFR 140 GFAS, University of Rennes 1, Faculty of Medicine, France \\ ${ }^{3}$ Department of Cytology, Institute of Zoology, Faculty of Biology, University of Warsaw, Poland
}

\begin{abstract}
MAP kinases of the ERK family play important roles in oocyte maturation, fertilization, and early embryo development. The role of the signaling pathway involving ERK5 MAP kinase during meiotic and mitotic M-phase of the cell cycle is not well known. Here, we studied the localization of the phosphorylated, and thus potentially activated, form of ERK5 in mouse maturing oocytes and mitotically dividing early embryos. We show that phosphorylation/dephosphorylation, i.e. likely activation/inactivation of ERK5, correlates with M-phase progression. Phosphorylated form of ERK5 accumulates in division spindle of both meiotic and mitotic cells, and precisely co-localizes with spindle microtubules at metaphase. This localization changes drastically in the anaphase, when phospho-ERK5 completely disappears from microtubules and transits to the cytoplasmic granular, vesicle-like structures. In telophase oocytes it becomes incorporated into the midbody. Dynamic changes in the localization of phospho-ERK5 suggests that it may play an important role both in meiotic and mitotic division. (Folia Histochemica et Cytobiologica 2011, Vol. 49, No. 3, 528-534)
\end{abstract}

Key words: mouse, oocyte, embryo, meiosis, mitosis, ERK5

\section{Introduction}

MAPK (Mitogen Activated Protein Kinase) signaling pathways are implicated in the regulation of a wide range of cellular processes such as proliferation, differentiation, and apoptosis [1]. Their function has been analyzed in various somatic cells [2-6], as well

Correspondence addresses: M.A. Ciemerych,

Department of Cytology, Institute of Zoology,

Faculty of Biology, University of Warsaw,

Miecznikowa Str. 1, 02-096 Warsaw, Poland;

e-mail: ciemerych@biol.uw.edu.pl

J.Z. Kubiak, Institute of Genetics \& Development,

CNRS-UMR 6061, Cell Cycle Group,

IFR 140 GFAS, University of Rennes 1,

Faculty of Medicine, France;

e-mail: jacek.kubiak@univ-rennes1.fr as in oocytes and embryos (e.g. [7, 8]). BMK1/ERK5/ /MAPK7 (Big MAP Kinase 1/Extracellular signal-Regulated Kinase 5/Mitogen Activated Protein Kinase 7; further called by us ERK5) is one of the less characterized MAP kinases of the ERK family. ERK5 knock-out mice proved that the major developmental function of this kinase is related to the placental, cephalic, hindgut, and, importantly, cardiovascular development [9-13]. Thus, mouse embryos lacking functional ERK5 gene failed to progress beyond E10.5, but until this developmental stage the cellular proliferation seemed unperturbed. Further analyses suggested, however, that ERK5 is activated at G2-M transition, and thus involved in the regulation of mitotic division [2,14-16]. Details of the mitotic function of this kinase have been analyzed in somatic cells, but have never been previously reported in oocytes or in embryonic cells. 
Among the MAP kinases family members studied so far in oocytes and embryos, only the function of ERK1 and ERK2 is well known. These two MAP kinases are activated by Mos-MEK1 pathway and are necessary for the establishment of CSF (CytoStatic Factor). CSF mediates the arrest of metaphase II oocytes in the mouse $[8,17]$ as well as in the frog [18]. Special attention has been paid to ERK1 and ERK2 MAPK pathway since it was shown to be necessary to sustain the meiotic arrest of maturing vertebrate oocytes, establishment of oocyte polarity, and impacting at the correct size of the polar body (e.g. [19-24]). In-gel MBP kinase assay performed using mouse oocytes and one-cell embryos proved that ERK1 and ERK2 activity is high exclusively in oocytes, but undetectable during mitotic divisions of early mouse embryos $[8,25]$. The same assay showed also that other MBP (myelin basic protein, i.e. MAPK substrate) phosphorylating kinases are active both in oocytes and mitotically dividing embryos $[8,26]$, suggesting a potential role for other MAP kinases in the regulation of early embryonic mitosis. ERK5 is likely to fulfill this role. The evidence supporting the idea of ERK5 involvement both in meiosis and mitosis comes from analyses of MAP kinases substrates. Phosphorylated form of one of them, i.e. p90 ${ }^{\text {Rsk1 }}$, was detectable in meiotically dividing mouse oocytes, when ERK1 and ERK2 are fully activated, but also during the first mitosis of mouse embryo, when these two ERK kinases remain inactive ([25], our unpublished data). Since $\mathrm{p} 90^{\mathrm{rsk} 1}$ was shown to be phosphorylated by ERK5 in somatic cells [3], it is likely that $\mathrm{p} 90^{\mathrm{rsk}}$ is activated by the same pathway during M-phase of oocytes and early embryos. ERK5-encoding mRNA was detected in unfertilized oocytes, and in preimplantation embryos up to blastocyst stage, suggesting that the protein could be present at that stage [7]. The presence of ERK5 protein in pig oocytes was shown using Western blotting. However, the precise localization analysis was lacking [27]. Similarly, Wang et al. presented Western blot analysis of preimplantation mouse embryos ( 3.5 days of development) and non-conclusive immunodetection of active and inactive forms of ERK5 [7].

In the current paper we show that ERK5 is present in mouse oocytes and early embryos, its activation is precisely regulated during each of the M-phases studied, and that its association with both meiotic and mitotic spindle is highly controlled during M-phase progression.

\section{Material and methods}

Ethical issues. All animal studies presented were approved by Local Ethics Committee No. 1 in Warsaw, Po- land according to European Union Council Directive 86/ /609/EEC of 24 November 1986. All animals were raised on the premises.

Collection and culture of oocytes at germinal vesicle stage. Two- to three-month-old $\mathrm{F}_{1}(\mathrm{C} 57 \mathrm{Bl} / 10 \times \mathrm{CBA} / \mathrm{H})$ female mice were injected intraperitoneally with $10 \mathrm{IU}$ pregnant mare serum gonadotrophin (PMSG; Folligon, Intervet, Netherlands) to stimulate the development of ovarian follicles. Between 48 and 52 hours later the females were killed by cervical dislocation. Fully grown oocytes arrested at prophase of the first meiotic division (germinal vesicle stage [GV]) were released from ovarian follicles. Oocytes were freed from cumulus cells by pipetting and then cultured for $2 \mathrm{~h}$ in M2 medium (medium M16 buffered with HEPES; [28]) containing bovine serum albumin (BSA; $4 \mathrm{mg} / \mathrm{ml}$ ). Oocytes were fixed and subjected to immunofluorescence at GV stage, at the GVBD (germinal vesicle breakdown), and at selected, subsequent stages of in vitro maturation.

Collection, parthenogenetic activation of ovulated oocytes and in vitro culture of one- and two-cell embryos, blastocysts. $\mathrm{F} 1$ females were superovulated by injection of $10 \mathrm{IU}$ of eCG and $10 \mathrm{IU}$ of hCG (Intervet, Netherlands) 48-52 hours apart. Ovulated oocytes were collected 17-18 hours after hCG injection and cumulus cells were removed with hyaluronidase (Sigma Aldrich, Germany, 300 units $/ \mathrm{ml}$ PBS). For the parthenogenetic activation, the oocytes were exposed to $8 \%$ ethanol (POCh, Poland) in M2 medium for 8 minutes at room temperature [29], washed carefully and cultured in M2. Only those oocytes that extruded polar bodies and formed pronuclei were used for further analyses. Starting at 14 hours after activation embryos were scored every 10 minutes to determine the timing of the nuclear envelope breakdown (NEBD) and then collected for further analyses at different times after NEBD. Fertilized twocell embryos were obtained from superovulated and mated females 49 hours after hCG. Embryos were collected and cultured in M2 medium. Starting from 49 hours after hCG, embryos were observed every 10 minutes to determine the timing of NEBD and collected for further analyses at different times after NEBD. Blastocysts were obtained from uteri of superovulated and mated females at 90 hours after hCG.

In vitro culture of murine $\mathrm{NIH} 3 \mathrm{~T} 3$ fibroblasts and $\mathrm{C} 2 \mathrm{C} 12$ myoblasts. NIH3T3 fibroblasts were cultured in Dulbecco's modified Eagle's medium supplemented (DMEM, Invitrogen) with $10 \%$ fetal calf serum (FCS) and antibiotics (Invitrogen) at $37^{\circ} \mathrm{C}$ in $5 \% \mathrm{CO}_{2}$. Media were supplemented with penicillin (100 units $/ \mathrm{ml})$ and streptomycin $(100 \mathrm{mg} / \mathrm{ml})$. Cells were fixed 48 hours after plating and processed for Western blotting or immunodetection of tubulin, ERK5, and phospho-ERK5 epitopes. C2C12 murine myoblasts cell line was obtained from the European Collection of Cell Cul- 
tures (ECACC no. 91031101, passage no. 13). Cells were cultured in high-glucose DMEM supplemented with $10 \%$ FCS and antibiotics at $37^{\circ} \mathrm{C}$ in $5 \% \mathrm{CO}_{2}$. Cells were fixed and processed for immunodetection of tubulin and phospho-ERK5 epitopes at day 2 after plating, i.e. at the proliferative stage.

Western blotting. Protein samples of NIH3T3 cells were subjected to electrophoresis in 9\% SDS-PAGE gels [30]. Separated proteins were transferred to nitrocellulose membranes (Hybond C, Amersham Biosciences) and probed with rabbit polyclonal primary antibodies either against ERK5 or phospho-ERK5 (Upstate Biotechnology, USA) at the dilution of 1:1,000. Next, $\alpha$-tubulin was immunodetected with mouse monoclonal antibody (Sigma Aldrich, Germany). Antigen-antibody complexes were revealed using alkaline phosphatase conjugated anti-rabbit or anti-mouse secondary antibody (diluted 1:20,000, Jackson ImmunoResearch, USA) in combination with Enhanced Chemifluorescence reagent (ECF; Amersham Biosciences). Signal quantification was performed using ImageQuant 5.2 software (Amersham Biosciences).

Immunofluorescence. NIH3T3 cells and $\mathrm{C} 2 \mathrm{C} 12$ myoblasts plated and cultured on glass coverslips were fixed in $3.7 \%$ paraformaldehyde in PBS for $10 \mathrm{~min}$ at room temperature and permeabilized with $0.1 \%$ Triton X100 in PBS for 5 min. Oocytes and embryos were fixed in $3.7 \%$ paraformaldehyde in PBS for $30 \mathrm{~min}$ at room temperature and permeabilized with $0.1 \%$ Triton X100 in PBS for 5 min. Non-specific binding was blocked by $1 \mathrm{~h}$ long treatment with 3\% BSA in PBS. Rabbit polyclonal antibodies against phospho-ERK5 (Upstate Biotechnology, USA) were used at the dilution of 1:200 and the incubation was performed overnight at $4^{\circ} \mathrm{C}$. Next, $\alpha$-tubulin was immunodetected with mouse monoclonal antibody (Sigma Aldrich, Germany) followed by FITC-labeled anti-rabbit and TRITC-labeled anti-mouse antibodies (Jackson ImmunoResearch, USA). Chromatin was visualized either with chromomycin A3 (Sigma Aldrich, Germany) diluted 1:1.000 in PBS with $5 \mathrm{mM} \mathrm{MgCl}_{2}$ for $0.5 \mathrm{~h}$ at room temperature or propidium iodide present in mounting medium (Vector Laboratories, USA). Samples incubated in chromomycin A3 were mounted in Citifluor (Citifluor Ltd, UK) on glass slides. Immunofluorescence analysis was accompanied by appropriate negative controls, i.e. samples were not subjected to the primary antibodies, but instead they were incubated in 3\% BSA in PBS. All samples were examined with laser scanning confocal microscope (Axiovert 100M, Carl Zeiss Jena, Germany) and processed using Adobe Photoshop 6.0.

\section{Results and discussion}

Since ERK5 is known to be phosphorylated upon M-phase in human cells [2,16,31], and its role in mam- malian meiosis is virtually unknown, we decided to begin characterization of this MAP kinase in mouse oocytes by following the dynamics in its phosphorylation state using a specific anti-phospho-ERK5 (P-ERK5) antibody.

In germinal vesicle $(\mathrm{GV})$ stage oocytes (meiotic prophase I) phosphorylated ERK5 is absent from the $\mathrm{GV}$ and is detected as a background staining within the cytoplasm (Figure 1, GV). Following M-phase entry, upon germinal vesicle breakdown (GVBD), the P-ERK5 signal becomes detectable close to condensing bivalents (Figure 1, GVBD $+1 \mathrm{~h}$ ). This staining increases with the progression of the prometaphase and reflects organization of microtubules forming meiotic spindle around the individualized bivalents. The intensity of the staining is highest in the vicinity of the chromosome bivalents (Figure 1, GVBD $+3 \mathrm{~h}$ ). In metaphase of the first meiotic division P-ERK5 localizes entirely to the spindle microtubules, and the background, i.e. cytoplasmic staining, becomes negligible (Figure 1, metaphase I). At the time of the first polar body extrusion, at the anaphase I/telophase I transition, P-ERK5 is no longer detectable on the spindle microtubules. However, it remains clearly positioned next to the telophase groups of chromatin and within the midbody. Concomitantly the decrease in the cytoplasmic staining is observed (Figure 1, telophase I). During the metaphase of the second meiotic division P-ERK5 staining becomes again localized exclusively to the meiotic spindle, as it was during the metaphase I (Figure 1, metaphase II). At this stage the cytoplasmic staining is also reduced to a minimum. Thus, the P-ERK5 localization in oocytes undergoing meiotic maturation shows that active form of ERK5 MAP kinase localizes specifically to the meiotic spindle. The affinity of ERK5 to the spindle microtubules manifests very early upon GVBD and persists during the long metaphase I which takes about 8 hours. The progressive incorporation of ERK5 to the spindle is manifested by its total disappearance from the cytoplasm when the meiotic spindle is fully assembled both in metaphases I and II. Microtubuleassociated localization of other MAP kinases, and some of their activators, has been reported. MEK1/2 kinase, which activates ERK1/2, was shown to be limited to the meiotic spindle, and its active form was shown to be located within the midbody during anaphase [32,33]. On the other hand, ERK1/2 MAP kinases were detectable at the spindle poles. ERK3 was also recently reported to co-localize to spindle microtubules, although the activation of this kinase was not investigated [34]. The localization and activation of ERK5 in mouse oocytes and early embryos has not been precisely studied to date; but, its expres- 


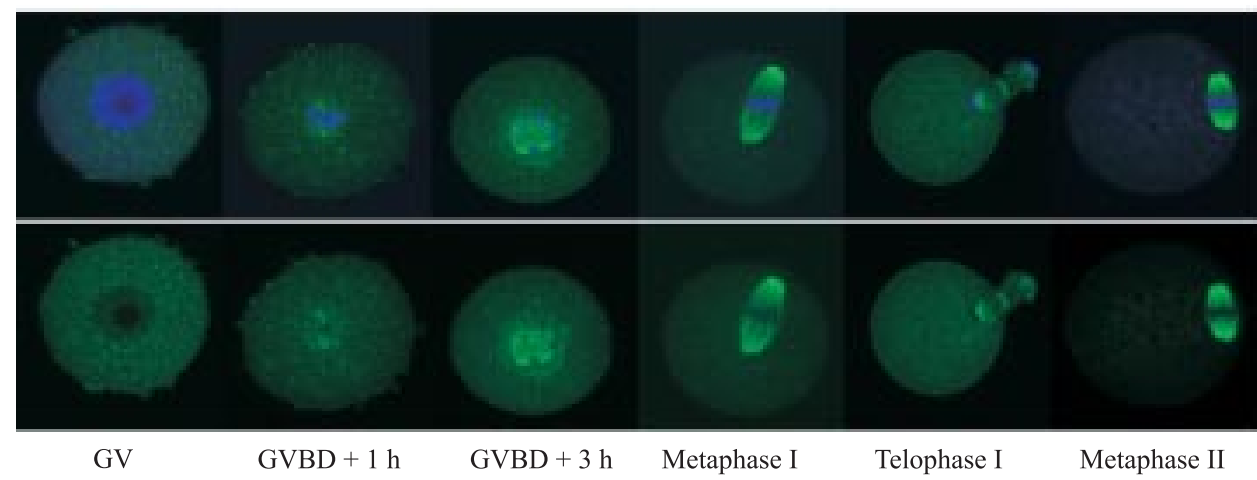

Figure 1. P-ERK5 localization in maturing mouse oocytes. Oocytes were analyzed at GV stage, and then during the formation of the metaphase plate and spindle of the first meiotic division (1 and $3 \mathrm{~h}$ after GVBD), at metaphase I (5 h after GVBD), telophase I (8 h after GVBD) and at metaphase II (9 h after GVBD). Top row - immunolocalization of P-ERK (green) and chromatin visualization (blue), overlay; bottom row — immunolocalization of P-ERK (green)

A

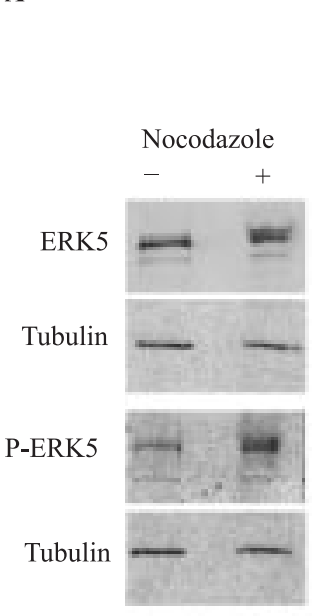

B

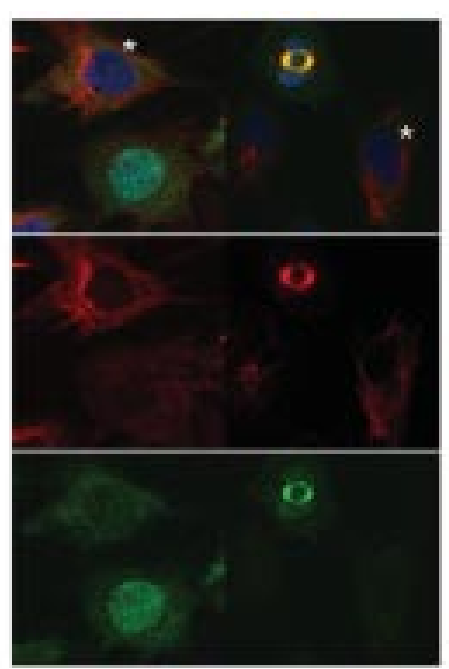

Prophase

M-phase
C

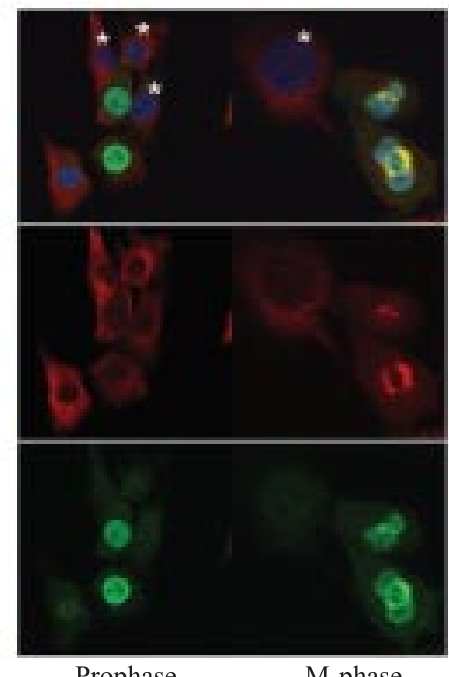

Figure 2. P-ERK5 in murine NIH3T3 fibroblasts and C2C12 myoblasts. A. Immunoblotting of ERK5 and P-ERK5 in lysates of asynchronously growing and nocodazole synchronized NIH3T3 fibroblasts. Note the presence of upper, slowmigrating, i.e. phosphorylated form of ERK5 in nocodazole treated cells. Immunodetection of $\alpha$-tubulin was performed to show the equal loading of the analyzed samples; B. Immunolocalization of P-ERK5 in asynchronously growing NIH3T3 fibroblasts; C. Immunolocalization of P-ERK5 in asynchronously growing C2C12 myoblasts. NIH3T3 and C2C12 cells were fixed in interphase, prophase and metaphase. Top row - immunolocalization of P-ERK5 (green), $\alpha$-tubulin (red), and visualization of chromatin (blue), overlay; middle row - immunolocalization of $\alpha$-tubulin (red); bottom row immunolocalization of P-ERK5 (green). Note the P-ERK accumulation in prophase nuclei and spindle and chromosome localization in metaphase. White asterisks indicate interphase nuclei lacking P-ERK5 staining

sion was shown in pig oocytes [27], and mouse preimplantation embryos [7]. However, images of ERK5 and P-ERK5 localization in mouse blastocysts presented by Wang et al. showed high and uniform signal within the cytoplasm of inner cell mass and trophectoderm cells [7], clearly different from that in the oocytes studied by us. This prompted us to analyze the P-ERK5 localization also in preimplantation mouse embryos.
To verify the specificity of the antibody used, we performed Western blotting and immunolocalization analyses of total ERK5 and P-ERK5 in murine somatic NIH3T3 cells. Similarly as presented by others (e.g. $[2,16])$, we detected a clear increase in the phosphorylated, up-shifted form of ERK5 in nocodazolesynchronized, i.e. mitotic cells. This result was obtained using both anti-total ERK5 (Figure 2A, ERK5) and anti-P-ERK5 antibody (Figure 2B, P-ERK5). 
A

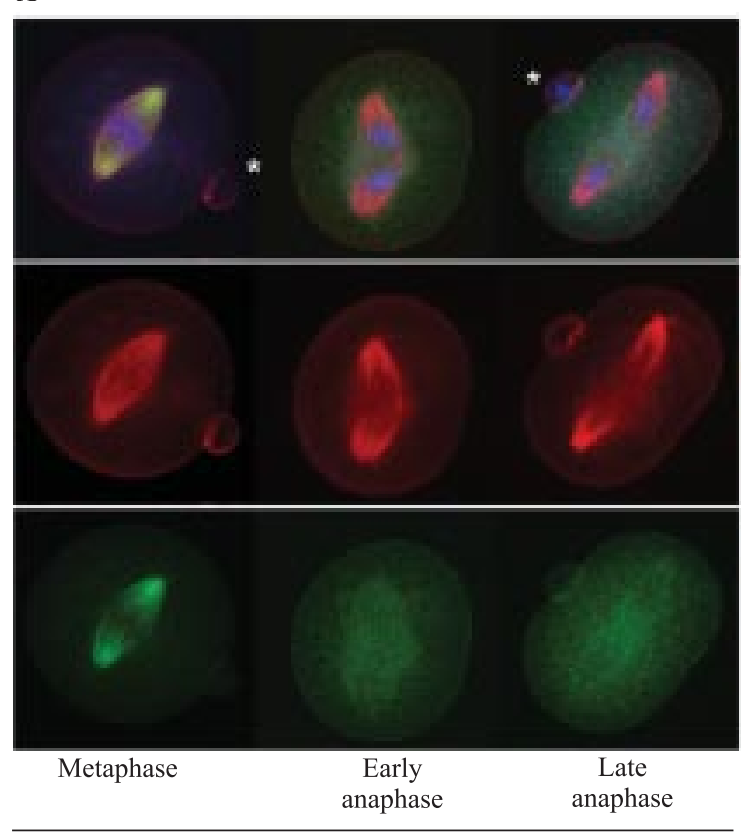

One-cell embryo
B

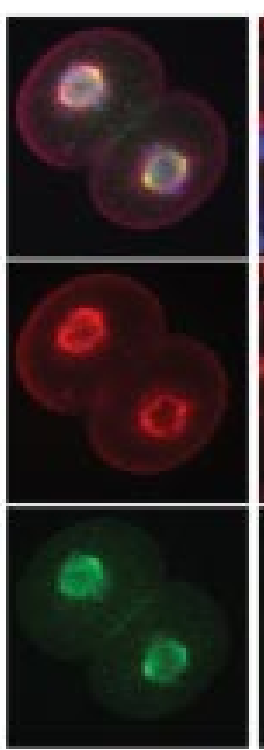

Metaphase

Two-cell embryo
C

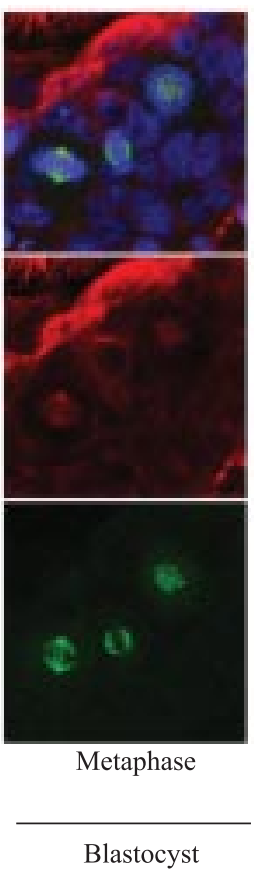

Figure 3. P-ERK5 localization in preimplantation mouse embryos. A. One-cell mouse parthenogenetic embryos fixed at metaphase and anaphase of first mitotic division. B. Two-cell mouse embryo fixed at metaphase of the second mitotic division. C. Mitotically dividing inner cell mass in blastocyst. Top row — immunolocalization of P-ERK5 (green), $\alpha$-tubulin (red), and visualization of chromatin (blue), overlay; middle row — immunolocalization of $\alpha$-tubulin (red); bottom row - immunolocalization of P-ERK5 (green)

Importantly, the phosphorylated form of ERK5 localized not only to the meiotic spindles, as described above, but also to the mitotic spindle, as shown in mouse NIH3T3 cells (Figure 2B) and C2C12 myoblasts (Figure 2C). In the interphase NIH3T3 and $\mathrm{C} 2 \mathrm{C} 12$ cells, a low cytoplasmic staining is detectable, while the nuclei are entirely devoid of P-ERK5 (Figures $2 \mathrm{~B}$ and $2 \mathrm{C}$, white asterisks). The nuclear localization of P-ERK5 becomes clear during prophase (Figure 2B and C, prophase). Further, in M-phase, the P-ERK5 staining is restricted to the mitotic spindle only in NIH3T3 cells (Figure 2B, M-phase), while in murine myoblasts $\mathrm{C} 2 \mathrm{C} 12 \mathrm{P}-\mathrm{ERK} 5$ colocalizes not only to the spindle, but also to chromosomes (Figure 2C, M-phase). Thus, Western blotting and also immunolocalization confirm that the signal detected in murine oocytes corresponds to the phosphorylated form of ERK5.

To perform precise analysis of P-ERK5 during $\mathrm{M}$-phase progression in embryonic cells we studied the well-characterized model of mitotically dividing mouse one-cell embryos. The first mitotic division of mouse embryo can serve, due to its duration of approximately 120 minutes, as a useful tool to study the mitotic events [35-39]. This feature allowed us to precisely analyze the localization and dynamics of ERK5 activation in M-phase. At metaphase of the first mi- totic division of one-cell embryos, similarly as was shown for oocytes, P-ERK5 perfectly colocalized to the spindle microtubules (Figure 3A, metaphase). Moreover, the cytoplasmic staining was not detected.

As soon as the anaphase started the pattern of P-ERK5 localization changed dramatically. First of all, the P-ERK5 signal was lost from the microtubules, and became visible as a diffused cloud around the spindle (Figure 3A, early anaphase). This signal dispersed during the progression to late anaphase and beginning of cytokinesis (Figure 3A, late anaphase). Concomitantly, the cytoplasmic staining increased (Figure 3A, early and late anaphase). Thus, following the metaphase-anaphase transition, P-ERK5 is no longer present on spindle microtubules, but seems to disperse around the anaphase spindle and further within the cytoplasm. Similar localization of P-ERK5 within the mitotic spindle was observed in two cell stage blastomeres (Figure 3B), and at further stages of preimplantation development (Figure 3C).

These results show that: 1) ERK5 is phosphorylated upon M-phase entry in meiosis and mitosis; 2 ) in both cases it localizes to the metaphase spindle microtubules; 3) and disappears from the spindle in early anaphase. Such localization suggests that ERK5 plays a similar function in spindle assembly/function in oocytes, embryos and somatic cells. 
ERK5/ BMK1/MAPK7 belongs to the subfamily of the MAP kinase family in mammals and it has some characteristic features: a large $\mathrm{C}$-terminus and a unique loop sequence [40]. Mammalian ERK5 has been shown to be activated by oxidative stress, hyperosmolarity, and several growth factors, including epidermal growth factor and nerve growth factor [40-45]. The mitotic function of ERK5 was suggested by the results of experiments focusing on both non-transformed cells such as fibroblasts and transformed cell lines such as HeLa cells. In HeLa cells, the expressing of constitutively active MEK5, and as a result the activation of ERK5, leads to an increase in the number of mitotic cells [2]. On the other hand, the use of the dominant negative form of ERK5, or siRNA suppressing ERK5 expression reduced mitotic index of analyzed cells [2]. Moreover, siRNA depletion of ERK5 decreases cell survival of mitotically dividing cells [14]. However, the fact that mouse embryos lacking functional ERK5 gene are able to survive until approximately E10.5 strongly suggests that ERK5 function can be replaced by another enzyme. Other MAP kinases, like ERK1 and ERK2, are candidates for such a function. Despite the fact that localization of ERK1 and ERK2 does not precisely reflect the P-ERK5 position within the mitotic spindle, it is well known that these kinases play a crucial role in meiotic spindle organization and function [8]. Analyses of oocytes from mice deficient in Mos kinase, an upstream ERK1/2 activating kinase, proved that ERK1/2 regulate microtubule dynamics $[17,19$, 20]. Recent discoveries of ERK3 localization and function in mouse oocytes identify a new candidate, which together with ERK1/2 and ERK5, might be involved in the regulation of spindle dynamics [34]. ERK3 was shown to be crucial for successful mouse development, but again, similarly as it was described for ERK5 knock-out mice, ERK3 deficiency did not prohibit mitotic divisions of the vast majority of embryonic tissues [46]. This kinase, however, similarly to ERK1/2 and ERK5, has been shown to be phosphorylated during M-phase entry and dephosphorylated at metaphase/anaphase transition [47]. The ERK4-deficient mice also did not reveal any significant mitotic phenotype; moreover, these animals were not only viable but also fertile [48]. Thus, only the perturbation of the activity ERK1/2 MAP kinases pathway, as shown by Mos knock-out mice analyses, has fatal consequences for the mouse fertility impacting at the oocyte maturation $[17,49,50]$. Mos knockout does not influence the mouse embryo development and animal viability probably due to ERK1 and ERK2 activation via Ras/Raf pathway [51]. However, it is possible that other MAPK, such as ERK5, may also act to rescue Mos deficiency following fertilization. Further studies will be necessary to solve this issue.

\section{Acknowledgements}

JZK was supported by a grant from ARC (4900) and LCC.

\section{References}

1. Rubinfeld H, Seger R. The ERK cascade as a prototype of MAPK signaling pathways. Methods Mol Biol. 2004;250:1-28.

2. Cude K, Wang Y, Choi HJ et al. Regulation of the G2-M cell cycle progression by the ERK5-NFkappaB signaling pathway. J Cell Biol. 2007;177:253-264.

3. Ranganathan A, Pearson GW, Chrestensen CA, Sturgill TW, Cobb MH. The MAP kinase ERK5 binds to and phosphorylates p90 RSK. Arch Biochem Biophys. 2006;449:8-16.

4. Cameron SJ, Abe J, Malik S, Che W, Yang J. Differential role of MEK5alpha and MEK5beta in BMK1/ERK5 activation. J Biol Chem. 2004;279:1506-1512.

5. Roberts OL, Holmes K, Muller J, Cross DA, Cross MJ. ERK5 is required for VEGF-mediated survival and tubular morphogenesis of primary human microvascular endothelial cells. J Cell Sci. 2010;123:3189-3200.

6. Roberts OL, Holmes K, Muller J, Cross DA, Cross MJ. ERK5 and the regulation of endothelial cell function. Biochem $S o c$ Trans. 2009;37:1254-1259.

7. Wang Y, Wang F, Sun T et al. Entire mitogen activated protein kinase (MAPK) pathway is present in preimplantation mouse embryos. Dev Dyn. 2004;231:72-87.

8. Verlhac MH, Kubiak JZ, Clarke HJ, Maro B. Microtubule and chromatin behavior follow MAP kinase activity but not MPF activity during meiosis in mouse oocytes. Development. 1994;120:1017-1025.

9. Yan L, Carr J, Ashby PR, Murry-Tait V, Thompson C, Arthur JS. Knockout of ERK5 causes multiple defects in placental and embryonic development. BMC Dev Biol. 2003;3:11.

10. Regan CP, Li W, Boucher DM, Spatz S, Su MS, Kuida K. Erk5 null mice display multiple extraembryonic vascular and embryonic cardiovascular defects. Proc Natl Acad Sci USA. 2002;99:9248-9253.

11. Hayashi M, Kim SW, Imanaka-Yoshida K et al. Targeted deletion of BMK1/ERK5 in adult mice perturbs vascular integrity and leads to endothelial failure. J Clin Invest. 2004;113: 1138-1148.

12. Sohn SJ, Sarvis BK, Cado D, Winoto A. ERK5 MAPK regulates embryonic angiogenesis and acts as a hypoxia-sensitive repressor of vascular endothelial growth factor expression. J Biol Chem. 2002;277:43344-43351.

13. Hayashi M, Lee JD. Role of the BMK1/ERK5 signaling pathway: lessons from knockout mice. J Mol Med. 2004;82:800-808.

14. Girio A, Montero JC, Pandiella A, Chatterjee S. Erk5 is activated and acts as a survival factor in mitosis. Cell Signal. 2007;19:1964-1972.

15. Inesta-Vaquera FA, Campbell DG, Arthur JS, Cuenda A. ERK5 pathway regulates the phosphorylation of tumour suppressor hDlg during mitosis. Biochem Biophys Res Commun. 2010;399:84-90.

16. Inesta-Vaquera FA, Campbell DG, Tournier C, Gomez N, Lizcano JM, Cuenda A. Alternative ERK5 regulation by phosphorylation during the cell cycle. Cell Signal. 2010; 22:1829-1837.

17. Verlhac MH, Kubiak JZ, Weber M et al. Mos is required for MAP kinase activation and is involved in microtubule organization during meiotic maturation in the mouse. Development. 1996;122:815-822. 
18. Haccard O, Sarcevic B, Lewellyn A et al. Induction of metaphase arrest in cleaving Xenopus embryos by MAP kinase. Science. 1993;262:1262-1265.

19. Araki K, Naito K, Haraguchi S et al. Meiotic abnormalities of c-mos knockout mouse oocytes: activation after first meiosis or entrance into third meiotic metaphase. Biol Reprod. 1996;55:1315-1324.

20. Choi T, Fukasawa K, Zhou R et al. The Mos/mitogen-activated protein kinase (MAPK) pathway regulates the size and degradation of the first polar body in maturing mouse oocytes. Proc Natl Acad Sci USA. 1996;93:7032-7035.

21. Verlhac MH, Lefebvre C, Kubiak JZ et al. Mos activates MAP kinase in mouse oocytes through two opposite pathways. Embo J. 2000;19:6065-6074.

22. Kosako H, Gotoh Y, Nishida E. Mitogen-activated protein kinase kinase is required for the mos-induced metaphase arrest. J Biol Chem. 1994;269:28354-28358.

23. Nishiyama T, Ohsumi K, Kishimoto T. Phosphorylation of Erp1 by p90rsk is required for cytostatic factor arrest in Xenopus laevis eggs. Nature. 2007;446:1096-1099.

24. Inoue D, Ohe M, Kanemori Y, Nobui T, Sagata N. A direct link of the Mos-MAPK pathway to Erp1/Emi2 in meiotic arrest of Xenopus laevis eggs. Nature. 2007;446;1100-1104.

25. Kalab P, Kubiak JZ, Verlhac MH, Colledge WH, Maro B. Activation of p90rsk during meiotic maturation and first mitosis in mouse oocytes and eggs: MAP kinase-independent and dependent activation. Development. 1996;122:1957$-1964$.

26. Yan C, Luo H, Lee JD, Abe J, Berk BC. Molecular cloning of mouse ERK5/BMK1 splice variants and characterization of ERK5 functional domains. J Biol Chem. 2001;276:1087010878.

27. Pelech S, Jelinkova L, Susor A et al. Antibody microarray analyses of signal transduction protein expression and phosphorylation during porcine oocyte maturation. J Proteome Res. 2008;7:2860-2871.

28. Fulton BP, Whittingham DG. Activation of mammalian oocytes by intracellular injection of calcium. Nature. 1978; 273:149-151.

29. Cuthbertson KS. Parthenogenetic activation of mouse oocytes in vitro with ethanol and benzyl alcohol. J Exp Zool. 1983; 226:311-314.

30. Laemmli UK. Cleavage of structural proteins during the assembly of the head of bacteriophage T4. Nature. 1970; 227:680-685.

31. Zen K, Yasui K, Nakajima T et al. ERK5 is a target for gene amplification at $17 \mathrm{p} 11$ and promotes cell growth in hepatocellular carcinoma by regulating mitotic entry. Genes Chromosomes Cancer. 2009;48:109-120.

32. Yu LZ, Xiong B, Gao WX et al. MEK1/2 regulates microtubule organization, spindle pole tethering and asymmetric division during mouse oocyte meiotic maturation. Cell Cycle. 2007;6:330-338.

33. Sun SC, Xiong B, Lu SS, Sun QY. MEK1/2 is a critical regulator of microtubule assembly and spindle organization during rat oocyte meiotic maturation. Mol Reprod Dev. 2008; 75:1542-1548.

34. Li S, Ou XH, Wang ZB et al. ERK3 is required for metaphaseanaphase transition in mouse oocyte meiosis. PLoS One. 2010;5:e13074.
35. Sikora-Polaczek M, Hupalowska A, Polanski Z, Kubiak JZ, Ciemerych MA. The first mitosis of the mouse embryo is prolonged by transitional metaphase arrest. Biol Reprod. 2006;74:734-743.

36. Ciemerych MA, Maro B, Kubiak JZ. Control of duration of the first two mitoses in a mouse embryo. Zygote. 1999;7: 293-300.

37. Maciejewska Z, Polanski Z, Kisiel K, Kubiak JZ, Ciemerych MA. Spindle assembly checkpoint-related failure perturbs early embryonic divisions and reduces reproductive performance of LT/Sv mice. Reproduction. 2009;137:931-942.

38. Kubiak JZ, Ciemerych MA, Hupalowska A, Sikora-Polaczek $\mathrm{M}$, Polanski Z. On the transition from the meiotic to mitotic cell cycle during early mouse development. Int J Dev Biol. 2008;52:201-217.

39. Teperek-Tkacz M, Meglicki M, Pasternak M, Kubiak JZ, Borsuk E. Phosphorylation of histone $\mathrm{H} 3$ serine 10 in early mouse embryos: Active phosphorylation at late S phase and differential effects of ZM447439 on first two embryonic mitoses. Cell Cycle. 2010;9:56-69.

40. Lee JD, Ulevitch RJ, Han J. Primary structure of BMK1: a new mammalian map kinase. Biochem Biophys Res Commun. 1995;213:715-724.

41. Abe J, Kusuhara M, Ulevitch RJ, Berk BC, Lee JD. Big mitogen-activated protein kinase 1 (BMK1) is a redox-sensitive kinase. J Biol Chem. 1996;271:16586-16590.

42. Chao TH, Hayashi M, Tapping RI, Kato Y, Lee JD. MEKK3 directly regulates MEK5 activity as part of the big mitogenactivated protein kinase 1 (BMK1) signaling pathway. $J$ Biol Chem. 1999;274:36035-36038.

43. Zhou G, Bao ZQ, Dixon JE. Components of a new human protein kinase signal transduction pathway. J Biol Chem. 1995; 270:12665-12669.

44. Kato Y, Tapping RI, Huang S, Watson MH, Ulevitch RJ, Lee JD. Bmk1/Erk5 is required for cell proliferation induced by epidermal growth factor. Nature. 1998;395:713-716.

45. Kamakura S, Moriguchi T, Nishida E. Activation of the protein kinase ERK5/BMK1 by receptor tyrosine kinases. Identification and characterization of a signaling pathway to the nucleus. J Biol Chem. 1999;27426563-26571.

46. Klinger S, Turgeon B, Levesque K, Wood GA, Aagaard-Tillery $\mathrm{KM}$, Meloche S. Loss of Erk3 function in mice leads to intrauterine growth restriction, pulmonary immaturity, and neonatal lethality. Proc Natl Acad Sci USA. 2009;106:16710-16715.

47. Tanguay PL, Rodier G, Meloche S. C-terminal domain phosphorylation of ERK3 controlled by Cdk1 and Cdc14 regulates its stability in mitosis. Biochem J. 2010;428:103-111.

48. Rousseau J, Klinger S, Rachalski A et al. Targeted inactivation of Mapk4 in mice reveals specific non-redundant functions of Erk3/Erk4 subfamily MAP kinases. Mol Cell Biol. 2010;30:5752-5763.

49. Hashimoto N, Watanabe N, Furuta $\mathrm{Y}$ et al. Parthenogenetic activation of oocytes in c-mos-deficient mice. Nature. 1994; 370:68-71.

50. Colledge WH, Carlton MB, Udy GB, Evans MJ. Disruption of c-mos causes parthenogenetic development of unfertilized mouse eggs. Nature. 1994;37065-68.

51. Yamauchi N, Kiessling AA, Cooper GM. The Ras/Raf signaling pathway is required for progression of mouse embryos through the two-cell stage. Mol Cell Biol. 1994;14:p6655-6662. 